\title{
Figure-ground perception and random geometry
}

H. L. FRISCH AND B. JULESZ ${ }^{1}$

BELL TELEPHONE LABORATORIES

Constructs of random geometry were applied to the problem of figure-ground perception. Random-dot images of black and white dots with various area fractions and tesselations (square and triangular lattices) were used as stimuli. The constructs of random geometry are correlation functions of $n$-th order and some functionals defined on them. The only parameter which is independent of the tesselation used is the first-order correlation which is the area fraction. It was first conjectured and then experimentally verified that figureground perception is not affected by the various tesselations used. Thus, figure-ground phenomena depend only on the area fraction of the white and black dots in the stimulus. There is a perceptual bias for white, that is, figure-ground reversal is easiest at 40 percent white-black area fraction. It was also experimentally shown that size-constancy prevails in figure-ground perception, but brightness-constancy does not.

\section{Perception of ambiguous figures.}

An essential characteristic of visual perception is the very fact that ambiguous figures can be perceived in only one way at a time. These ambiguous stimuli can be organized into various percepts by the central nervous system (CNS) and the selection of a percept at a given time depends on some organizational factors. The presence or absence of a given organizational factor in the possible percepts of an ambiguous figure will determine the probability of experiencing the various percepts. Thus using ambiguous stimuli is a powerful method of determining and rank ordering the factors which operate in perceptual organization.

The most important and basic ambiguous stimulus class is based on the perception of figure-ground. The perceptual dichotomy between figure and ground is always present. Under usual conditions the figure contains much more organizational factors than the ground and no ambiguity is experienced. The "figure" in this phenomenon does not mean necessarily the form of a recognizable object, but rather refers to a definite perceptual change which occurs when two domains which have a common boundary are viewed. Whichever is seen as sharp, definite, having a "thing" character is called the figure, while the rest is perceived as some "substance" and is the ground. The more identical the organizational factors are in the two percepts the more easy it is to reverse them perceptually. These figureground reversals were extensively studied by Rubin (1915) and were used by the Gestaltist school in formulating their well known "rules" of perceptual organization (Rubin, 1958).

These rules of perceptual organization can be rank ordered and the five most important ones are as follows:
(1) Proximity and similarity. Spatially adjacent units of similar brightness, color and shape form connected clusters. This cluster perception is not only fundamental in shape recognition, but is fundamental in texture discrimination as well and was further clarified in a recent study (Julesz, 1962).

(2) Area. The smaller a closed region the more it tends to be seen as a figure (see Fig. 1). The complement holds as well; the larger the area of a region is the more it appears to be the ground.

(3) Closedness. Areas with closed contours tend to be seen more likely as figure than do areas with open contours.

(4) Symmetry. The more symmetrical a closed region is, the more it appears as figure. The greater the number of symmetries the region possesses increases this tendency.

(5) Smooth continuation. From many possible perceptual organizations those tend to be perceived, which will minimize changes or interruptions in the contours of the perceived constituents.

These principles have an intuitive appeal and can be easily verified; nevertheless they are loosely defined notions and vary in their generality. The clustering due to proximity and similarity and smooth continuation are more explicitly organizational principles than the rest, which can be regarded rather as factors of organization. In figure-ground reversals the figure and ground share a common boundary, therefore the factors of area and closedness cannot be separately chosen, but effect simultaneously the two organizations. Some factors, like symmetry, are very suitable for mathematical treatment but are very weak and insignificant compared to area or closedness, which are much stronger cues. This is shown in Figs. $2 a$ and $2 b$ where the principles of closedness and area override symmetry.

\section{Mathematical freatment of percepfual organization.}

In the forthcoming discussion we restrict ourselves to figure-ground reversals and postpone the more general problem of perceptual reversals in ambiguous

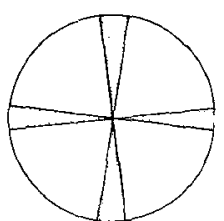

(a)

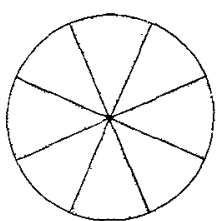

(b)

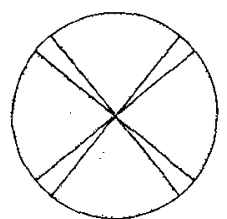

(c)
Fig. 1. The role of area in figure-ground perception. The smaller area tends to be perceived as figure, thus in (a) the figure is a wheel with black spokes, in (c) a wheel with white spokes and (b) is ambiguous. 


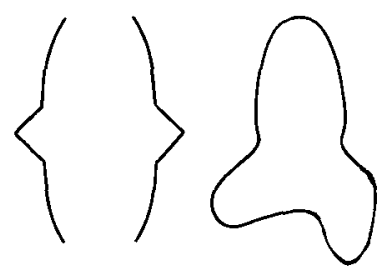

(a)

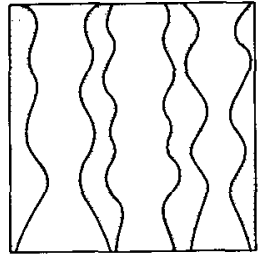

(b)
Fig. 2. Demonstration that the rules of closedness and area override the importance of symmetry. In (a) the open symmetric drawing tends less to be perceived as the figure than the closed asymmetric drawing. In (b) the symmetric white stripes are less often perceived as the figure than the asymmetric stripes of smaller areas.

figures. In order to avoid the complex familiarity cues, which interact in uncontrollable ways, we used random textures with computer-controlled statistical and geometrical properties.

Within the above restrictions on figure-ground reversal we tried to test the main factors underlying this phenomenon by a systematic mathematical treatment. We used methods of random geometry which had several advantages. First, many parameters in random geometry (also referred to as geometrical probability theory or statistical geometry or in part integral geometry) have an intuitive psychological appeal and can be easily related to the Gestaltist rules of organization. Second, many of the invariances of visual perception such as unchanged perception of figures under translation, expansion-contraction and rotation are inherently invariant for constructs in integral geometry. Many constructs of the more general random geometry can be applied to media with arbitrary invariances and can be derived by randomly throwing certain simple objects of various shapes and sizes (such as lines, triangles, polygons) on figures and determining the statistics of the intersections. This is analogous to some neurophysiological findings in the visual cortex of cat and monkey (Hubel \& Wiesel, 1962), according to which receptive fields of increasing complexity and of great density are extracting features from the input stimuli.

Before we pursue with this method, some questions have to be clarified. One limitation of integral geometry for studying visual perception is its inherent invariancy under rotation. This means that only a restricted class of perceptual problems can be treated by this method, which are perceptually unchanged under rotation of the stimulus. Recognition of faces or reading the alphabet are known perceptual tasks which are not invariant under rotation. Figure-ground reversal of random textures is obviously rotation-invariant and the use of integral geometry is justified and we do not have to employ the more general stochastic processes of random geometry.

Another limitation is the finite number of receptors in the visual system. This discreteness of sampling is trivial in integral geometry, but results in great difficulties for other treatments.
An interesting problem is raised by size constancy. By size constancy we mean that the precepts, or "observables," remain invariant under geometrical transformations which expand or contract the input figure, within very wide limits. For continuous transformations of this type an obvious choice of "observables" is one which is isomorphic with certain Lie derivatives (Lie group germs) and some attempts were made recently to regard them as preprocessors in the visual system (Hoffman, 1966). On the other hand, the visual system is sometimes viewed as being approximated, in structure and function, by a discrete, parallel, automaton described by Unger $(1958,1959)$. The essence of this automaton is that a large but finite number of computational modules are simultaneously instructed by a master module (which may be "distributed" among the other modules) and each module can communicate only with its nearest neighbor modules. This machine can recognize and discriminate among many two- or onedimensional patterns, using only a few instructions. We were interested in studying the combination of these two views of the visual system. By extending the concept of the Lie derivative to a finite difference operator we attempted to find algorithms for an Unger machine which would enable it to recognize and match to memory templates linear patterns (linear so that we need not be bothered by considering rotations) by carrying out the extended group operations (translations, contractions and expansions). These linear patterns are onedimensional blocks of zeros and ones. While suitable Unger machine programs could be found which carried out translations and contractions or translations and expansions, we as well as Professor Unger were unable to find a program which was an algorithm for carrying out translations and contractions as well as expansions as group operations. Basically the difficulty arises that in expanding blocks of adjacent zeros and ones an overlap of the blocks occurs, which is not obtained on contraction. We thus conjecture that the parallel automaton view and the Lie germ preprocessor view of the visual system are incompatible, one or the other or both may have to be abandoned. We believe that a more general, in some sense globally connected machine is required for carrying out the Lie group operations under which the "Gestalt" is to remain invariant.

It should be noted again that these problems of perceptual invariances, which are the mathematical descriptions of the Gestalt rule of symmetry, are really secondary problems. There are several perceptual factors such as area or closedness which are much more influential, and in this study we give more attention to these. Nevertheless, some experimental and theoretical treatment of these invariances will be included.

\section{A hierarchy of hypotheses for figure-ground reversal.}

We wish to determine which geometrical factors most strongly affect figure-ground reversal and later more general perceptual reversals in ambiguous figures. We have already pointed out the "all or none" character of 
these reversals which remind one of the "phase transitions" or "order-disorder" phenomena studied by the physical chemist when dealing with cooperative assemblies. We employ computer-generated random textures as stimuli. These planar random textures (or media) constitute mathematically certain stochastic processes -with a two-dimensional vector parameter, $\underline{r}$, lying within the plane of the texture whose components are referred to a suitable, preselected coordinate system. Loosely speaking, the totality of what constitutes the random texture, i.e., the stochastic process, is given by an infinite hierarchy of suitable probability distribution functions containing all the geometric information about the texture distribution functions.

We will employ two-phase random textures composed of white regions of area fraction ( $\emptyset$ ) and black regions of area fraction $(1-\emptyset)$; more specifically, regular tesselations of the plane such as the square and triangular in which the individual squares or triangles are colored black or white according to a binomial distribution. The hierarchy of probability distributions in those cases refers to the random (Poisson distribution) throwing of polygons of $n$ vertices, $\{n\}=\left(\underline{r}_{1}, \underline{r}_{2}, \ldots, \underline{r}_{n}\right)$, $\mathrm{n} \geq 1$ ( $\mathrm{n}=1$ a point, $\mathrm{n}=2$ a line, $\mathrm{n}=3$ a triangle, etc.) and asking with what probability, $\gamma_{n}(\{n\})=\gamma_{n}$, thesen vertices all lie in the white region of the texture. This definition of the $\gamma_{n}$ 's is a generalization of the classical Buffon needle problem which dealt with the determination of a $\gamma_{2}$ (Frisch \& Stillinger, 1963). The statistical geometry is contained, so to say, in the hierarchy $\gamma_{1}, \gamma_{2}$, $\ldots$, and specific geometrical properties can be expressed naturally by certain functionals of the ${ }_{n}{ }_{n} s$ in ways which will be illustrated below (Frisch \& Stillinger, 1963; Frisch, 1965; Novikoff, 1962).

It appears therefore possible that the response function of subjects viewing our ambiguous random textures and perceiving a figure-ground reversal, $R(\emptyset)$, is a functional $F$ of $\gamma_{n}(\{n\})=\gamma_{n}$ (where the $\gamma_{m}(\{m\})$ with $m<n$ are obtained from $\gamma_{n}(\{n\})$ by excizing $(n-m)$ vertices of the $n-$ gon; e.g., $\gamma_{n-1}(\{n-1\})$ is $\gamma_{n}\left(\underline{r}_{1}, \ldots, \underline{r}_{n-1}\right.$, $\left.\underline{r}_{n}=\underline{r}_{n-1}\right)$. This functional may involve certain parameters such as one (or more) scale lengths $L$, if size constancy is not maintained, and certain intrinsic psychological parameters, as could describe a bias of black over white, $f$, etc. We thus obtain immediately a hierarchy of, in principle, testable hypotheses

$$
\begin{aligned}
& R(\emptyset)=F^{(R)}\left(\gamma_{n}(\{n\}) ; L, f\right) \\
& =F_{n}(R) \quad ; n=1,2, \ldots,
\end{aligned}
$$

depending on the least value of $n$ which suffices to represent the experimental results. This approach is strictly valid only for textures of infinite extent. The $100 \times 100$ arrays used in this study can be assumed to approximate sufficiently the infinite case.

Hypothes is 1 states that figure-ground reversal depends only on $\gamma_{1}$ the random throwing of a point and for the textures, used by us, is identical with $\emptyset$, the area fraction of the white regions. $R(\phi)$ could then be given merely by a Heaviside function or some smoothed version of argument $\emptyset-1 / 2(1-f)$ (cf., Fig. 3 ). If this is the case, $R(\emptyset)$ should be "independent" of $\gamma_{n}$ for $n \geq 2$. A sufficient but not necessary condition is that $R(\phi)$ remain invariant under a change of type of tesselation, say from square to triangular, providing $\varnothing$ in these tesselations is kept fixed. Such a change of tesselation, with $\emptyset$ fixed, varies all $\gamma_{n}$ for $n \geq 2$. Hypothesis 1 will be tested by psychological experiments as described below. If Hypothesis 1 is valid, since $\emptyset$ does not depend on sample array size, any measured dependence on lattice size is because of some psychological factor rather than that reflected in the size dependence of $\gamma_{n}$ for $n \geq 2$. Nevertheless, before we test experimentally this hypothesis let us discuss the mathematical and psychological meaning of some other hypotheses.

Hypothes is 2: Figure-ground reversal depends only on $\mathrm{Y}_{2}$. (For an isotropic random medium $\gamma_{2}$ is only a function of the distance $r=\left|\underline{r}_{2}-\underline{r}_{1}\right|$ of the directed line segment, $\underline{r}_{2}-\underline{r}_{1}$.) At this level one can distinguish between random textures with long-range order and those which possess only short-range order (since $\gamma_{2} \rightarrow \phi^{2}$ for indefinitely large $\mathbf{r}$ only in the latter case). A melting or sublimation of a solid (crystal) to yield a fluid is an example of a physical chemical phase transition involving a loss of long-range order; on the other hand, the vapor-liquid critical point phase transformation involves no such change. Whether long-range order in a texture is perceived by our visual system is an interesting speculation for which evidence either pro or con could be obtained using certain controlled computergenerated random textures in which both kinds of ordering is suitably varied.

Restricting ourselves to short ranged correlations only we could have the case that a reversal depends on the mean perimeter per unit area, $\langle\ell / a\rangle$. This entity is related to the initial slope of the angular average of $\gamma_{2}, \overline{\gamma_{2}}$ and we could call it Hypothesis $2 a$, the lower case letter following the 2 indicating that less information than is provided in the whole function $\gamma_{2}$ is necessary to frame the hypothesis. The fact that the initial slope of $\bar{y}_{2}$ is proportional to $\langle\ell / a\rangle$ can

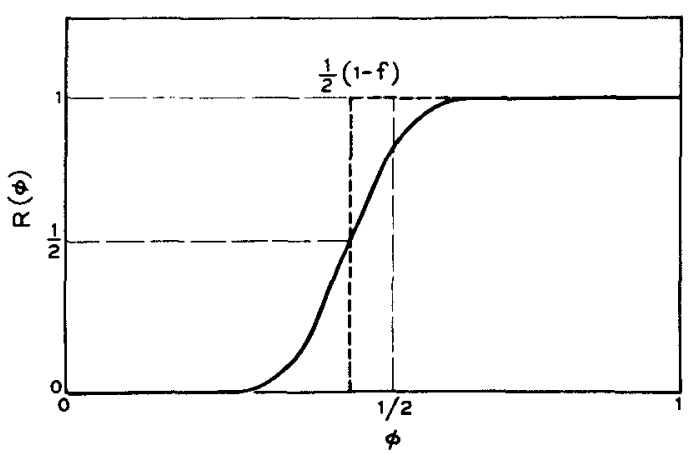

Fig. 3. Possible response function under hypothesis 1. Curve-.Heaviside function of argument $\phi-1 / 2(1-f)$, curve - - A smoothed version of the same function. 
be heuristically seen: By marginal consisteney $\gamma_{2}$ is linearly dependent on the probability that the line segment $r$ has end points lying in two differently colored regions. Thus the differential $\mathrm{d} \mathrm{Y}_{2}$ must be proportional to the probability that an "infinitesimal'i ruler, $\mathrm{d} \underline{\text { }}$, averaged over all orientations, crosses the periphery of, say, the black region, which is proportional to $\langle\ell / a\rangle$.

For the random square tesselation with unit length of a square, and $|\underline{r}|<1, \underline{r}=(x, y)$,

$$
\gamma_{2}(r)=\emptyset^{2}+\emptyset(1-\emptyset)(1-|x|)(1-|y|) \text {. }
$$

Angular averaging followed by differentiation yields:

$$
\langle\ell / a\rangle=4 \emptyset(1-\emptyset)
$$

This expression can be derived in an easier way: In some position on an infinite square lattice consider a square formed from $n^{2}$ unit areas bounded by $2 n^{2}$ unit segments. In order that a segment, say a vertical segment, be part of the perimeter, the square cell to the left and right must be of different phases, either black, white, or white, black; either of which is realized with probability $\emptyset(1-\emptyset)$. Hence

$$
\langle\ell / a\rangle=\frac{2 n^{2} \cdot 2 \phi(1-\emptyset)}{n^{2}}=4 \phi(1-\emptyset) .
$$

Let the reversability of figure-ground be proportional to $\langle\ell / a\rangle$, or a monotonic function of $\langle\ell / a\rangle$. (See Fig. 4.) Thus the larger $4 \varnothing(1-\emptyset)$, the easier it becomes to reverse figure and ground. We can also postulate that the smaller $\langle\ell / a\rangle$, the easier it is to perceive white cluster: as figure on a black background, and the closer $\langle\ell / a\rangle$ approaches unity, the easier it becomes to perceive black clusters as figure on a white background.

The same calculations can be undertaken for a triangular lattice. Figure 5 shows three types, of lines $\mathrm{A}, \mathrm{B}$ and $\mathrm{C}$, which form $\mathrm{N}$ triangles, each having an area of $\sqrt{3 / 4}$. There are $3 \mathrm{~N}$ sides, each of which is shared by two triangles. In order for a side to be part

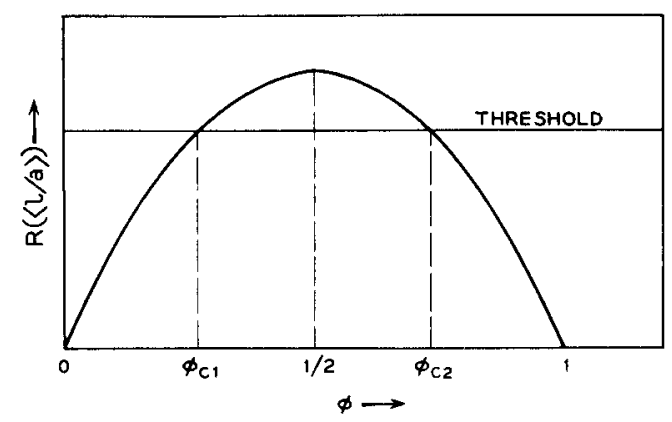

Fig. 4. Possible response function under hypothesis 2 a. Ordinate is proportional to $</ \mathbf{a}>$. Bias $\mathbf{f}$ has been taken to be zero. The region of ambiguity extends from $\phi_{\mathrm{c} 1} \leq \emptyset<\boldsymbol{\sigma}_{\mathrm{c} 2}$ and varies with the nature of the tesselation, if the threshold is fixed.

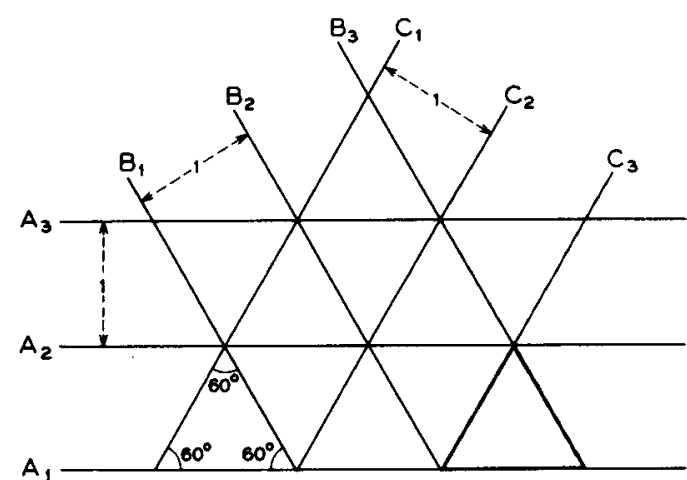

Fig. 5. The three sets of lines $A, B, C$ which form the triangular lattice.

of the perimeter, the triangles containing this side must belong to different phases. The probability that they do is $2 \varnothing(1-\emptyset)$. This is the fraction of distinct sides (counted not twice) of the triangles which are part of the perimeter. Thus

$$
\langle\ell / a\rangle=\frac{\frac{3}{2} \mathrm{~N} 2 \phi(1-\emptyset)}{N \sqrt{3 / 4}}=4 \sqrt{3 \phi(1-\emptyset) .}
$$

A similar calculation can be carried out for the hexagonal lattice, which can be regarded as being generated from six adjacent triangular cells repeated periodically. For this case:

$$
\langle\ell / a\rangle=\frac{3.2 \emptyset(1-\emptyset)}{\frac{6 \sqrt{3}}{4}}=\frac{4}{\sqrt{3}} \emptyset(1-\emptyset)
$$

Thus, the triangular lattice has a $\langle\ell / a\rangle$ which is $\sqrt{3}$ times larger than the value for the square lattice and this is $\sqrt{3}$ larger than the value for the hexagonal lattice. It is reasonable to assume that the psychological response would also change accordingly. Since a change of $\sqrt{3}$ is not negligible, it is reasonable to assume that the corresponding perceptual changes of figure-ground reversals for various tesselations can be measured.

It is interesting to note that $\langle\ell / a\rangle$ is not invariant under dilation. On the other hand, $\langle l / \sqrt{a}\rangle$ is invariant. This shows again the flexibility of random geometrical methods. It is relatively easy to construct parameters which either exhibit certain invariances or not, depending solely on the psychological findings to be modelled.

Hypothesis $3 a$. We assume that the convex dispersal of phase 1 in phase 2 has an effect on figure-ground reversal. This assumption is psychologically reasonable since the convexity or concavity of figures can be effortlessly perceived. The convex dispersal of a phase in another one can be expressed as a functional of $\gamma_{3}$, but can be again computed from some elementary considerations. Using the mathematical definition of convexity we throw at random rulers of length $2 \ell, 2 \ell<<1$, and ask that the points 0 and $2 \ell$ belong to the same 
phase but the point $\ell$ (in the center of the rulers) to the other phase. Since three distinct points are involved, this probability can be computed from $\gamma_{3}$. Consider Fig. 6 for a square lattice. For $2 l<<1$, this can only occur if the center of the ruler lies in a square 2 whose phase is different from squares 1 and 3 in which must lie the points 0 and $2 \ell$, respectively. For any angle $\theta$, the probability of points $0, \ell, 2 \ell$, lying in phases black, white, black is $\left(\ell^{2} / 2\right) \sin \theta \cos \theta \emptyset^{2}(1-\emptyset)$, i.e., 4 times the area of the triangle $A B C$ divided by 4 unit areas, since the location of the point $\ell$ can be chosen equally well to be in squares $1,2,3$ or 4 . Averaging over all angles gives simply $\langle\sin \theta \cos \theta\rangle=\langle\sin 2 \theta\rangle=\frac{1}{\pi}$. Thus the angle averaged probability of $\mathrm{P}_{\mathrm{W}, \mathrm{B}, \mathrm{W}}(\ell)$ is

$$
P_{W B W}(\ell)=\frac{1}{2 \pi} \ell^{2} \emptyset^{2}(1-\emptyset), 2 \ell<<1
$$

Similarly, since white goes into black when we interchange $\emptyset$ and $1-\emptyset$,

$$
\mathrm{P}_{\mathrm{BWB}}(\ell)=\frac{1}{2 \pi} \ell^{2} \phi(1-\phi)^{2}, 2 \ell<<1 .
$$

Now we note that if all dispersed clusters of a given phase were simply connected convex domains, then

$$
\begin{aligned}
a & =\lim _{\ell \longrightarrow 0} \frac{P_{W B W}(\ell)-P_{B W B}(\ell)}{\ell^{2}} \\
& =\frac{1}{2 \pi}\left[\phi^{2}(1-\emptyset)-\not(1-\phi)^{2}\right]
\end{aligned}
$$

would be positive and equal to $(1 / 2 \pi) \phi^{2}(1-\not)$ if white convex domains only were dispersed in black, and negative and equal to $(1 / 2 \pi) \emptyset(1-\emptyset)^{2}$ if black convex domains only were dispersed in white. In general, if the figureground reversal would depend only on convex dispersal then $\alpha(\phi)$ would be a measure of the perceptual response.

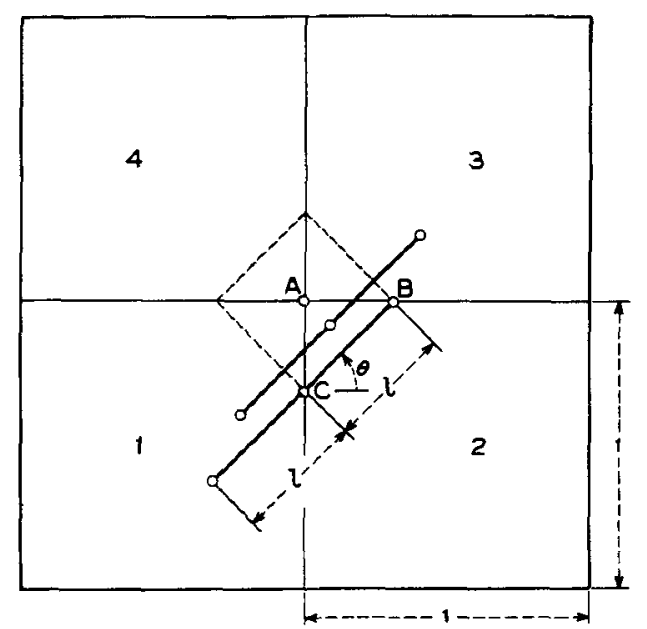

Fig. 6. Diagram illustrating the computation of $\alpha(\phi)$ for the square lattice.

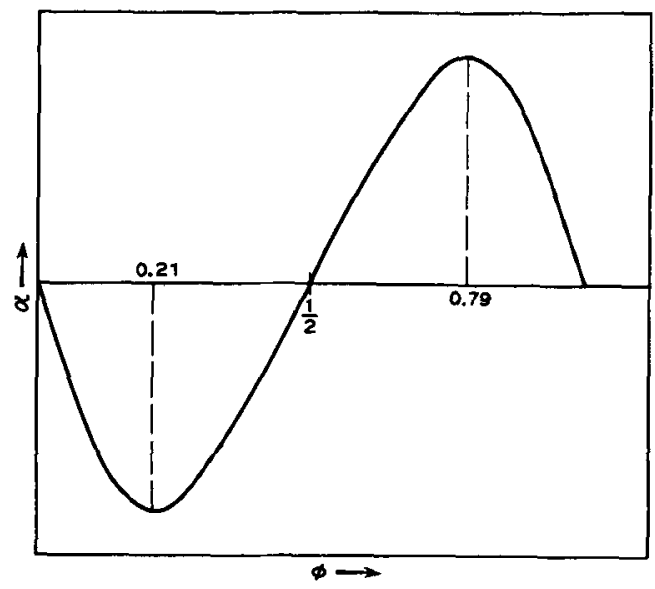

Fig. 7. The function $a(\varnothing)$ vs. $\emptyset$.

$\alpha(\phi)$ is plotted in Fig. 7. This function depends also on the class of tesselation used.

The more general criterion that one phase be dispersed in simply connected domains, whether convexor not, within the other (the multiply connected) phase is a functional of only $\gamma_{6}$. We shall not pursue this list of hypotheses further here. It should be clear from these examples how various specific geometrical properties can be formulated in terms of the ${ }_{n}{ }^{\prime} s$ which characterize the random texture.

\section{Experimental testing of the hypotheses.}

In order to verify experimentally the possible hypotheses the following straightforward yet cumbersome procedure could be followed. We could generate two classes of stimuli which would be identical in all their $\gamma_{n}$ correlation functions except for one $\gamma_{p}$, which would be different for the two classes. If figure-ground perception would be different for these two stimulus classes, Hypothesis $\mathrm{p}$ is accepted. That is, the perceptual response depends on the p-order correlation function alone. As we might try $p=1,2,3, \ldots$ we could successively exhaust all those higher-order correlation functions or some functionals defined on them which affect perception.

This lengthy procedure can be overcome, if we can verify that Hypothesis 1 is invariant for different tesselations. As we have already discussed, only the firstorder correlation $\gamma_{1}=\varnothing$ does not depend on the shape of the tesselation. We wanted to test experimentally whether figure-ground reversal does depend on various tesselations when the area fraction of black and white clusters is changed. If the perceptual response does not depend on the tesselation used we have verified that only $\emptyset$ is utilized in figure-ground perception, since all second or higher-order correlations are different for the various tesselations.

The experiments were conducted on 23 naive Ss, who were typists and technicians in our laboratory. The stimuli were viewed in two sizes. The larger series extended six degrees of arc, the smaller extended half 


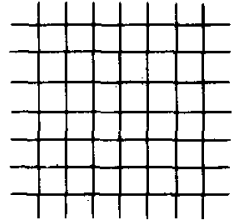

(a)

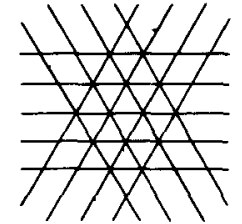

(b)

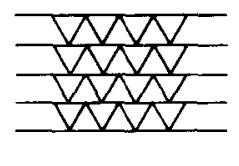

(c)
Fig. 8. Enlarged drawings of the types of tesselations used: (a) square, (b) triangular and (c) mixed triangular lattices.

this size. The stimuli used belonged to three tesselations, shown in Figs. $8 \mathrm{a}, 8 \mathrm{~b}$ and $8 \mathrm{c}$, respectively. In addition to the regular square lattice and the regular triangular lattice, a third configuration was used, which was obtained from the regular triangular tesselation by shifting horizontally by one cell every second row (see Fig. $8 \mathrm{c})$. The $\langle\ell / a\rangle$ of this tesselation is the same as that of the triangular lattice but $\gamma_{n}$ for $n \geq 2$ are all different. (Actually this configuration was obtained by a programming error.) Each of the images consisted of $10^{4}$ cells. For each tesselation 11 random-dot images were generated by a digital computer with area fractions $\emptyset=.25, .30, .35, \ldots, .70, .75$. Three typical samples for each tesselation are shown in Fig. 9. The checkerboard boundaries at the margins served to separate the random-dot images from the surround. Special care was exerted to reduce influences of areas outside this barrier by projecting these stimuli on freely suspended screens which were of the same size as the stimuli.

The Ss were instructed before the experiment to report within $5 \mathrm{sec}$. whether the stimulus was perceived as a black tabletop with white specks on it or as a white tabletop with black specks on it. If the $S$ was uncertain and could perceive it in both ways in temporal succession he could respond in three ways by reporting black ambiguous, ambiguous and white ambiguous, respectively (where the black ambiguous and white ambiguous indicated some preference). Thus, altogether five responses could be made, numbered from 0 to 4 . The Ss were told to make their judgments according to the perception of the table and not according to the estimation of the area fraction.

The 11 various area fractions were shown twice in different random order for the three tesselations. The same experiment was repeated for the half-reduced stimulus sizes. Thus, 132 judgments were made by each $S$ in a single test period.

The computer-generated stimuli were of high quality and contrast. In those experiments in which the brightness levels were kept constant a exposure meter of a field of view corresponding to the stimulus size was used.

\section{Results.}

As we have already discussed, each $\mathrm{S}$ could give four responses for each stimulus 0 (black), 1 (black ambiguous), 2 (ambiguous), 3 (white ambiguous) and
4 (white) referring to the ground (tabletop). Each of the three tesselations used contained 11 stimuli having various area fractions. The experiments were four times repeated; thus, for each stimulus and for each individual $\mathrm{S}$, an average response in the range between 0 and 4 was obtained. For each tesselation $t$ and each subject $s$ a response vector $R_{t s}$ was derived (having 11 components). As a next step, the Hamming distance between two tesselations and the same subject was introduced. For instance

$$
\begin{aligned}
\mathrm{D}_{\mathrm{s}}(\square, \Delta) & =\frac{1}{11}\left|\underline{\mathrm{R}}_{\square \mathrm{s}}-\underline{\mathrm{R}}_{\triangle \mathrm{s}}\right|= \\
& =\frac{1}{11} \sum_{\mathrm{n}=1}^{11}\left|\mathrm{R}_{\square \mathrm{sn}}=\mathrm{R}_{\triangle \mathrm{sn}}\right|
\end{aligned}
$$

is for subject $s$ the average response distance between the square lattice and the triangular lattice. Figure 10 shows this distance for 23 subjects. As the data indicates in average no subject made one unit response error (the maximum error could have been 4 units), but only a fraction of a unit. $D=\frac{1}{N} \sum D_{S}$ are summarized in Table 1 (where $\mathrm{N}$ is the number of subjects):

Table 1. The average response distance between pairs of different

$$
D=\frac{1}{N} \sum_{s=1}^{N} D_{s}\left(t_{i}-t_{i}\right)
$$

$D(\square, \Delta)$ (square, triangular) $=.315$

$D(\square, 0)$ (square, mixed triangular) $=.276$

$\mathrm{D}(\Delta, 0)$ (triangular, mixed triangular) $=.230$

ordinate is (NR/N4) 100 in percent, that is the psychological response averaged over all $\mathrm{N}$ subjects. The points $\square, \triangle$ and $O$ in Fig. 11 correspond to the means for the square, triangular and mixed triangular tesselations, respectively, whereas $-, \wedge, \cap$ correspond to the endpoints of the standard deviations for the three tesselations.

An inspection of Fig. 11 immediately reveals that for each tesselation the mean responses are within $\pm \sigma$. This is also an indication of why the acceptance of Hypothesis 1 is highly significant.

In this analysis the data collected for the large and small stimulus sizes were grouped together. In the next, analysis of variance was used to test whether size constancy existed for figure-ground perception. In the first series of experiments no corrections were made for the brightness increase as the projector lens was zoomed down for half size, therefore both size and brightness constancy were simultaneously tested. Table 2 shows the results for the 11 area fractions, where for each area fraction we continued the measurements for the three tesselations measured twice for 23 subjects; thus $3 \times 2 \times 23=138$ data points were obtained both for the small and large stimuli. 

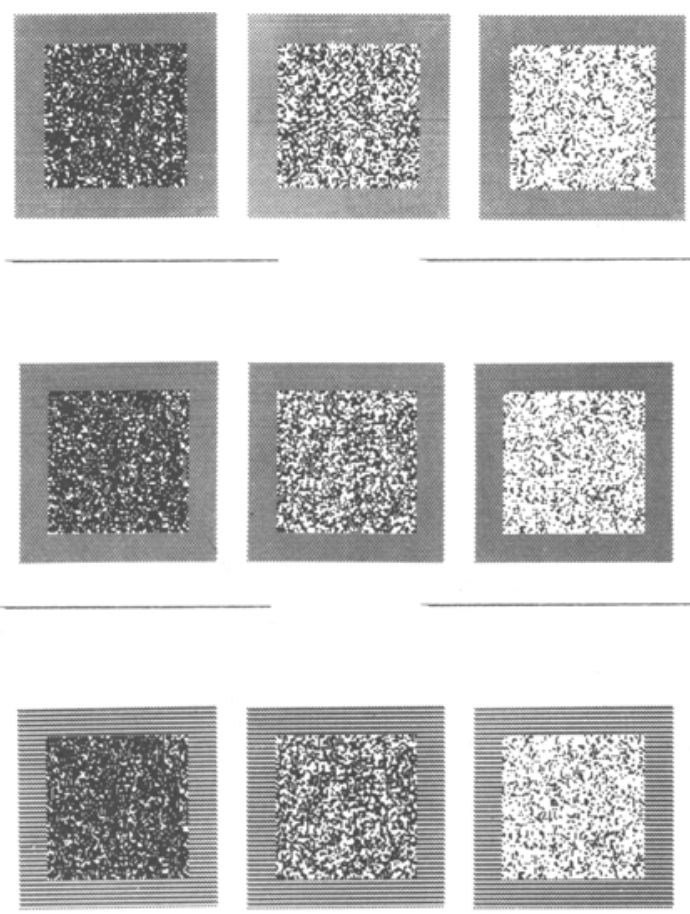

Fig. 9. Samples of stimuli used in the experiments. Left column, $\phi=0.25$; middle, $\phi=0.50$; and right, $\phi=0.75$ area fraction. Top, square tesselation; middle, triangular tesselation; bottom, mixed triangular tesselation.

The hypothesis that the large and small stimuli are perceived identically for figure-ground is only acceptable for $\phi \geq .60$. For $.25 \leq \emptyset<.60$, this hypothesis has to be rejected. Since $t$ is decreasing both for large and small $\emptyset$ values, it can be conjectured that for small values around .15 or less, the hypothesis might be acceptable again. It is interesting to note that size and brightness constancy holds for those area fractions which correspond to the most stable figure-ground perception. For area fractions which give ambiguous figure-ground perception-giving rise to figure-ground reversals-the constancy hypothesis fails. The greater the perceptual ambiguity the greater the level of significance that the constancy hypothesis fails.

This asymmetry in area fraction is further emphasized in Fig. 11 where the small and large stimuli are combined and separately plotted for the three tesselations. The psychological response function is asymmetrical and the half point is $\phi=0.42$ instead of 0.5 , and at $\phi=0.55$ the response corresponds to the dual obtained at $\emptyset=0.25$. This asymmetry $f$ is probably due to the psychological bias of using white paper and black ink more often than using a blackboard with white chalk.

Table 2 contains two more results. The combined average of all 23 subjects and all 11 area fractions is grouped together and is tested for significance. The $t$ test reveals that size and/or brightness constancy has to be rejected (highly significantly). The last result shows the same $t$ test on a subgroup of seven Ss, who gave the most consistent results. Even for this subgroup the constancy hypothesis had to be rejected, although it is interesting to note that the criterion of rejection was somewhat less significant. It seems as if $\mathrm{Ss}$ with better performance are exhibiting more perceptual constancy, although still not a full constancy

This unexpected departure from perceptual constancy led to an obvious experiment. We tried to separate brightness constancy from size constancy. The previous series of experiments were repeatedusing two stimulus sizes of 8 and 4 degrees of arc in width and height, except the brightnesses were kept constant. The eight best Ss who were most consistent and only the square tesselation were used. We extended the area fractions by two stimuli $(\phi=0.15$ and 0.20$)$ thus using 13 stimuli which were repeated four times in various orders for both stimulus sizes. Before testing, the entire range from 0.15 to 0.75 was demonstrated in monotonically increasing order. The results are given in Fig. 12.

Figure 12 indicates a strong size constancy for figure-ground perception when the brightness is kept constant. This implies that the departure from perceptual constancy in the previous experiments was due to the brightness changes. Thus size constancy prevails but brightness constancy does not. A detailed description of a t test for the 13 area fractions is given in the fourth section of Table 2. All experiments show size constancy to be very highly significant.

Because of size constancy, we plotted the averaged response function both for the small and large stimuli in Fig. 13. This diagram is very similar to Fig. 11, except that the range is extended and only one tesselation is used. The same tendency of increased standard deviation in the ambiguity regions is present in Fig. 13. Also, a strong perceptual bias $f$ is present in favor of white. The average response function $\left\langle\mathbf{R}_{\mathrm{S}}\right\rangle=1$ percent

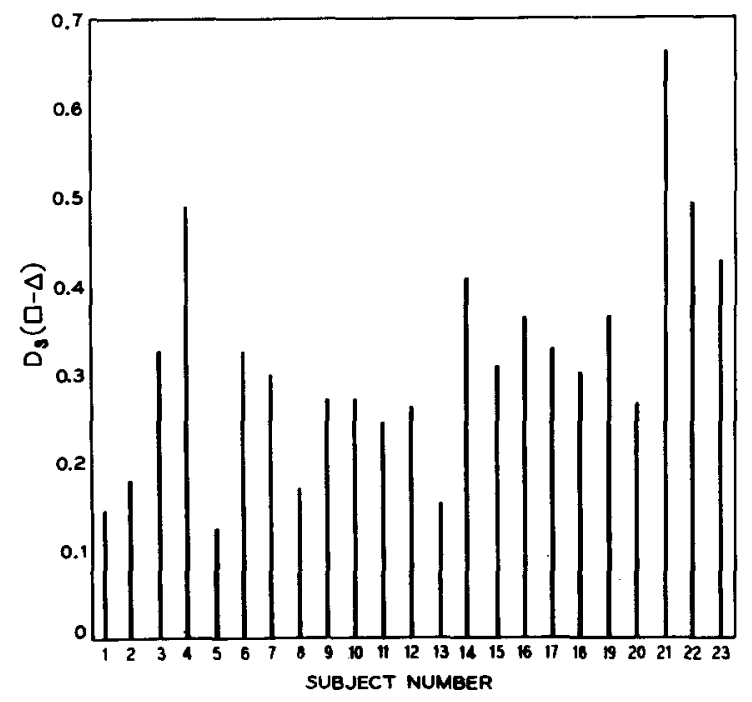

Fig. 10. Average response distance between the square and triangular lattice for 23 subjects. 


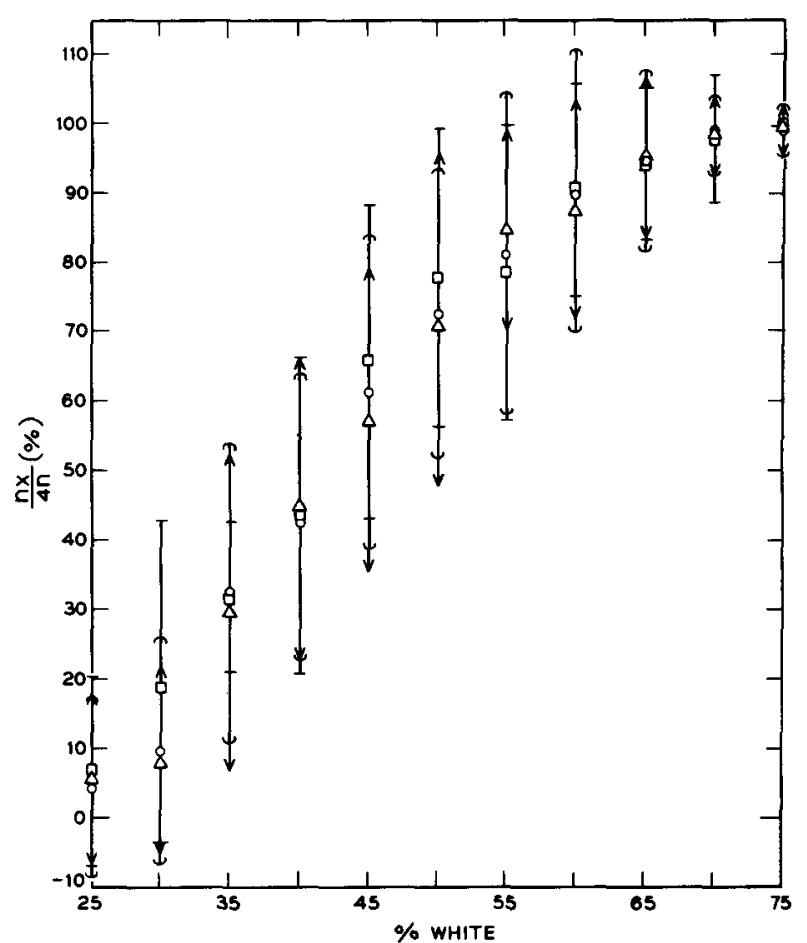

Fig. 11. Psychological response for the three tesselations used as a function of the area fraction.

Table 2. t-test of Brightness and Size Invariancy

\begin{tabular}{lccc} 
& & 1 & $\begin{array}{c}\text { tc } \\
\text { for } 99.9 \% \\
\text { confidence }\end{array}$ \\
\hline 23 Subjects & $25-75 \%$ & $* 10.763$ & 3.291 \\
\hline 7 Best of 23 Subjects & $25-75 \%$ & $* 5.648$ & 3.291 \\
\hline & $25 \%$ & $* 3.942$ & \\
& $30 \%$ & $* 3.947$ & \\
& $35 \%$ & $* 3.772$ & \\
& $40 \%$ & $* 4.279$ & \\
& $45 \%$ & $* 4.808$ & 3.370 \\
& $50 \%$ & $* 3.918$ & \\
& $55 \%$ & $* 3.469$ & \\
& $60 \%$ & 1.273 & \\
& $65 \%$ & 3.187 & \\
& $70 \%$ & 0.831 & \\
& $75 \%$ & 1.000 & \\
\hline & $15 \%$ & 0.000 & \\
& $20 \%$ & 1.800 & \\
& $25 \%$ & 2.260 & \\
& $30 \%$ & 0.721 & \\
(Brightness Compensated for) & $35 \%$ & 1.724 & \\
& $40 \%$ & 1.888 & 3.674 \\
& $45 \%$ & 0.769 & \\
& $50 \%$ & 1.800 & \\
& $55 \%$ & 2.295 & \\
& $60 \%$ & 1.686 & \\
& $65 \%$ & 1.800 & \\
& $70 \%$ & 1.000 & \\
& $75 \%$ & 0.000 & \\
\hline
\end{tabular}

*Values outside confidence limits indicates that practically all Ss perceived the tabletop as black. The same response function for perceiving the tabletop as black $\left(1-\left\langle R_{s}\right\rangle=1\right.$ percent) occurs not at $\phi=0.85$ (which is the complement of $\emptyset=0.15$ ) but at a much smaller $\phi=0.70$ value. The $\left\langle\mathrm{R}_{\mathrm{S}}\right\rangle=.50$ percent value corresponds to $\phi=0.38$ instead of $\emptyset=0.50$ which would be the case if no bias were present.

\section{Conclusions.}

Constructs of random geometry were applied to the problem of figure-ground perception. In order to avoid the influence of familiarity cues, random-dot images of black and white dots with various area fractions $\emptyset$ were used as stimuli. The constructs of random geometry are correlation functions of $n^{\text {th }}$ order, $\gamma_{n}$, and some functionals defined on them and depend on the tesselation of the stimulus. The only parameter which is independent of the tesselation used is the first-order correlation, which is the area fraction $\emptyset$. It was first conjectured and then experimentally verified that figureground perception is not affected by the various tesselations used. This also means that figure-ground phenomena depend only on the area fraction.

The experiments also showed that size constancy prevails in figure-ground perception for all area fractions. On the other hand, brightness constancy fails to hold for area fractions which result in figure-ground reversals. The psychological response is asymmetrical, biased by a factor $f$ in the direction of perceiving the ground as white. The maximum figure-ground reversal occurs at $\emptyset \approx 0.4$ instead of $\emptyset=0.5$, that is, for a stimulus which has 40 percent black clusters in a 60 percent white ground.

Although the psychological findings showed that figure-ground perception is based solely on the simplest possible stimulus parameter, the area fraction, the article discusses a systematic treatment which can be used for more complex perceptual phenomena. Randomdot stimuli can be generated which differ only in one $\gamma_{k}$ (or a functional of $\gamma_{k}$ ) whereas the rest is identical. Whether this difference can be perceived or not determines the perceptual importance of the geometrical parameter in question. A similar program was tried previously by one of us (B. J.) keeping the k-th order probability distribution functions different and the $(k-1)$ and lower order statistics identical for two random textures viewed side-by-side (Julesz, 1962). The difficulty of this procedure was that differences in only the first- and second-order distribution functions had simple perceptual meanings (such as tonal quality and granularity), whereas differences in third-order statistics could often not be perceived. It seemed very difficult mathematically to introduce some functionals on the third-order statistics which could have some intuitive geometrical meaning and could have been perceptually differentiated. The fact that the stochastic processes which were known to exhibit these properties were restricted to certain n-th order Markov processes 
(Rosenblatt \& Slepian, 1962) was a less important limitation. In the random geometrical approach the $n-$ th order probability distribution is replaced by $\gamma_{n}$, the $n$-th order correlation function. The $\gamma_{n}-s$ and the functionals defined on them have direct geometrical meanings such as perimeter-area fraction, convexdispersal, single connectedness and as such might be more appropriate parameters in perceptual studies than $n$-thorder probability distributions and some moments defined on them which lack immediate geometrical appeal.

We would like also to note that some recent neurophysiological findings in cat (Hubel \& Wiesel, 1962) and monkey indicated the existence of receptive fields of a hierarchical order of increasing complexity. These units include edge and slit detectors, corner detectors, etc. of all orientations and sizes and suggest the idea to regard them as the $\mathbf{n}$-gons of the generalized Buffon needle problem (Leibovic).

Regardless of whether the random-geometrical approach has any deeper relationship with neurophysiological structures, the attempt to treat visual perception by techniques which proved successful in explaining order-disorder transitions in molecular physics of cooperative phenomena might have some merits. ${ }^{2}$ Perhaps, the Gestalt phenomena of perception which demonstrate global properties so different from their local constituents are only quantitatively more complex than the cooperative phenomena of physical chemistry which are the macroscopic manifestations of simple

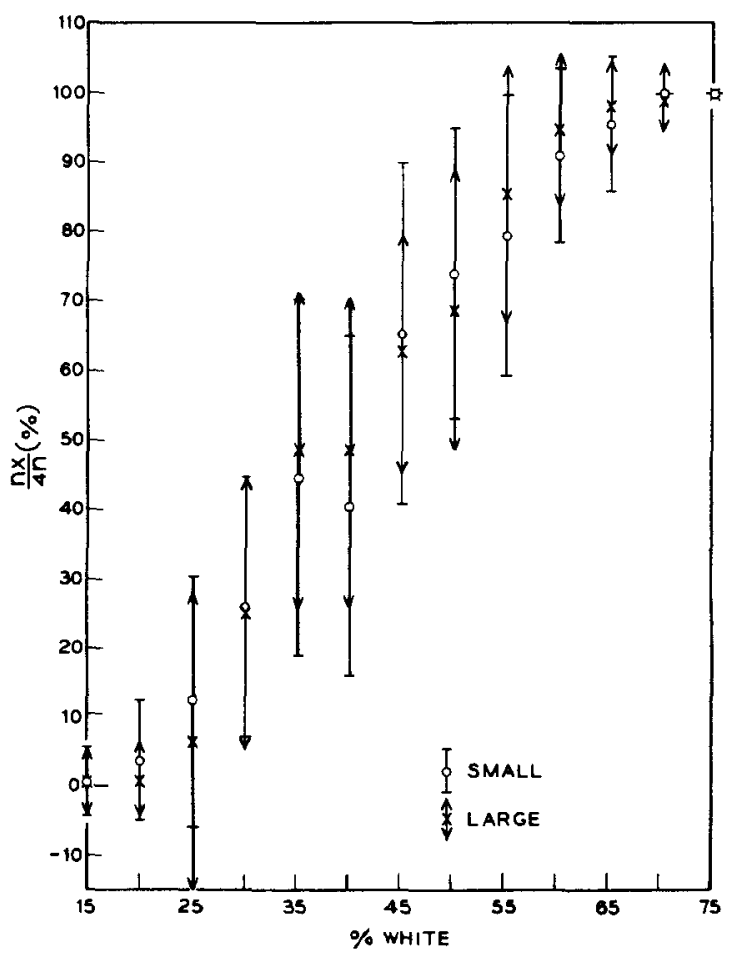

Fig. 12. Mean and standard deviation of the psychological response function for large ( 8 degree of arc) and small (4 degree of arc) stimuli.

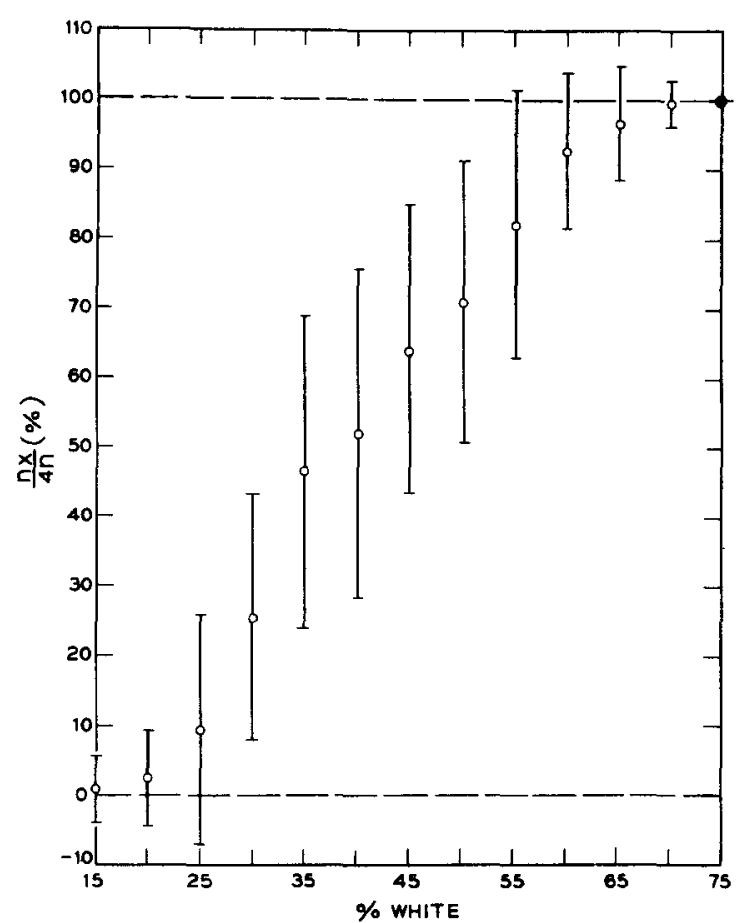

Fig. 13. Same as Fig. 12, except results for the large and small stimuli are summed.

rules and couplings between a large number of microscopic elements (Purcell, 1963).

\section{References}

Frisch, H. L. Statistics of random media. Trans. Soc. Rheology. 1965, 9, 293.

Frisch, H. L., \& Stillinger, F. H. Contribution to the statistical geometric basis of radiation scattering. J. Chem. Phys., 1963, $38,2200$.

Hoffman, W. C. The lie algebra of visual perception. J. math. Psychol, 1966, 3, No. 1, 65.

Hubel, D. H., \& Wiesel, T. N. Receptive fields, binocular interaction and functional architecture in the cat's visual cortex. J. Physiol., 1962, 160, 106.

Julesz, B. Visual texture discrimination, "Special issue on sensory information processing". IRE PGIT, Publ. IT-8, 1962. P. 84.

Leibovic, K. N. A model for information processing with reference to vision. (To be published.)

Novikoff, A. B. J. Integral geometry as a tool in pattern perception. In H. von Foerster and G. W. Fopf (Eds.), Principles of self organization. New York: Pergamon Press, 1962. P. 347.

Purcell, E. Parts and whole in physics. In D. Lemer (Ed.), Parts and wholes. New York: MacMillan, 1963. P. 11.

Rosenblatt, M., \& Slepian, D. $\mathrm{N}^{\text {th }}$ order Markov chains with any set of $\mathrm{N}$ variables independent. J. Soc. Indust. Appl. Math., $1962,10,537$.

Rubin, E. Figure and ground. In Beardslee and Wertheimer (Eds.), Readings in perception. Princeton, N. J.: Van Nostrand, 1958. P. 194. An excellent elementary review of Gestalt rules is given in J. Hochbert, Perception. Englewood Cliffs, N. J.: Prentice Hall, 1965.

Unger, S. H. A computer oriented toward spatial problems. Proc. IRE, $1958,46,1744$.

Unger, S. H. Pattern detection and recognition. Proc. IRE, 1959, $47,1737$. 


\section{Notes}

1. We thank Miss Ellen Gritz for developing the computer program which generated the displays and Mr. R. A. Payne and P. Farago for running the experiments.

2. In passing we would like to point out the possible utility of commencing an interdisciplinary effort by investigators such as we, whose individual disciplines (statistical machanics for HLF and experimental psychology for BJ) do not appear to overlap.
Individual reflection, followed by subsequent mutual discussion in which each could tutor the other on only specially relevant points of a special discipline, led to a mutual convergence on a well defined class of problems underlying this investigation, with an enhanced ability to participate felt by both investigators.

(Accepted for publication September 30,1966.) 